\title{
(In)segurança alimentar e nutricional: desenvolvimento de um indicador do problema e experimentação em famílias da Paraíba, Brasil
}

\author{
Food and nutrition (in)security: development of an indicator of the problem and \\ experimentation in families of Paraíba, Brazil
}

\section{(In)sécurité alimentaire et nutritionnelle: élaboration d'un indicateur du problème et les tests dans les familles de Paraíba, Brésil}

(In)seguridad alimentaria y nutricional: desarrollo de un indicador del problema y experiencia en familias de Paraíba, Brasil

\author{
Dixis Figueroa Pedraza* \\ (dixisfigueroa@gmail.com) \\ Márcia Cristina Sales** \\ (cristina.salles@yahoo.com.br)
}

Recebido em 29/12/2012/; revisado e aprovado em 23/02/2013; aceito em 17/03/2013

\begin{abstract}
Resumo: Apesar dos esforços, ainda não se conta com métodos que possam avaliar a (in)segurança alimentar em toda a sua extensão. Assim, a finalidade desse artigo é propor e aplicar uma medida complementar dos níveis de (in)segurança alimentar familiar medidos com a aplicação da Escala Brasileira de Insegurança Alimentar. A medição da severidade de insegurança alimentar possibilita identificar, entre os indivíduos com insegurança alimentar, aqueles que apresentam maior vulnerabilidade.

Palavras-chave: Insegurança alimentar. Nível de saúde. Condições sociais.
\end{abstract}

Abstract: Despite efforts, there are still no methods that can assess the food (in) security throughout its length. Thus, the purpose of this article is to propose and implement complementary measure levels of household food (in) security with the implementation of the Brazilian Food Insecurity Scale. Measuring the severity of food insecurity possible to identify, among individuals with food insecurity, those who are more vulnerable.

Key words: Food insecurity. Health status. Social conditions.

Résumé: Malgré les efforts déployés, pas encore réalisé avec des méthodes qui permettent d'évaluer la (in)sécurité alimentaire sur toute son extension. Ainsi, le but de cet article est de proposer et mettre en application une mesure complémentaire des niveaux de la (in)sécurité alimentaire de famille mesurée avec l'application de l'échelle brésilienne d'insécurité alimentaire. La mesure de la sévérité de l'insécurité alimentaire fournit la capacité d'identifier, parmi des personnes avec l'insécurité alimentaire, ceux qui présentent une plus grande vulnérabilité.

Mots-clés: Insécurité alimentaire. Niveau de santé. Conditions sociales.

Resumen: A pesar de los esfuerzos, el desarrollo de métodos que puedan evaluar la (in)seguridad alimentaria en toda su extensión no ha sido posible. Así, la finalidad de este manuscrito es proponer y aplicar una medida complementaria de los niveles de (in)seguridad alimentaria familiar con la aplicación de la Escala Brasileña de Inseguridad Alimentaria. La medición de la severidad de la inseguridad alimentaria posibilita identificar, entre los individuos con inseguridad alimentaria, aquellos que presentan mayor vulnerabilidad.

Palabras clave: Inseguridad alimentar. Nivel de salud. Condiciones sociales.

\section{Introdução}

A finalidade desse artigo é propor e aplicar uma medida complementar dos níveis de (in)segurança alimentar familiar medidos com a aplicação da Escala Brasileira de Insegurança Alimentar. Apesar dos esforços, não se conta, ainda, com métodos que possam dar conta de avaliar o fenômeno em toda a sua extensão. A importância do aperfeiçoamento dos métodos e indicadores para o monitoramento e a avaliação da situação de segurança alimentar vem sendo reforçada por diferentes entidades, como a Organização para Agricultura e Alimentação das Nações Unidas (FAO). No Brasil, a preocupação vem se fazendo presente em diferentes instâncias, como as Conferências Nacionais deSegurança Alimentar e Nutricional.

O fenômeno da Segurança Alimentar e Nutricional engloba vários aspectos: (a) um componente alimentar, que se refere à produção, disponibilidade, comercialização, acesso e consumo do alimento saudável e (b) um componente nutricional relacionado às práticas alimentares e à utilização biológica

\footnotetext{
* Universidade Estadual da Paraíba, Campina Grande, Paraíba, Brasil.

** Universidade Federal do Rio Grande do Norte, Nata, Rio Grande de Norte, Brasil.
} 
dos alimentos (indicadores antropométricos, bioquímicos, etc.) contemplando todas as fases do ciclo de vida e estendendo-se a grupos institucionalizados (creches, escolas, asilos, etc.) (PRADO et al., 2010). Assim, avaliar a Segurança Alimentar e Nutricional exige metodologias capazes de se aproximarem o máximo possível das múltiplas facetas do problema (PANELLI-MARTINS; SANTOS; ASSIS, 2008).

A caracterização do nível de segurança alimentar e nutricional é útil para a focalização das políticas sociais (URIBE; RESTREPO, 2008). Avaliar situações de risco é fundamental para a tomada de decisão em relação à necessidade de intervir, para quem, em que local, de que forma. Trata-se, portanto, de uma tarefa estratégica para definir prioridades de intervenção e dos seus impactos (PANELLIMARTINS; SANTOS; ASSIS, 2008).

Existem cinco métodos comumente utilizados para mensurar a segurança alimentar (PEREIRA; SANTOS, 2008). Quatro são métodos indiretos baseados nas repercussões de estados de insegurança alimentar no consumo de alimentos ou no estado nutricional: i) método da Organização das Nações Unidas para Agricultura e Alimentação; ii) pesquisas de despesas familiares, iii) avaliação de consumo de alimentos; e iv) avaliação antropométrica. $O$ único método para a medida direta de segurança alimentar é representado por uma escala fundamentada na experiência/percepção de insegurança alimentar (PEREIRA; SANTOS, 2008; PÉREZESCAMILLA; SEGALL-CORRÊA, 2008).

No Brasil, pesquisadores de várias instituições validaram um método para a avaliação direta da segurança alimentar, denominado Escala Brasileira de Insegurança Alimentar (EBIA) (FAVARO et al., 2007). Essa escala tem sido reconhecida como indicador sensível para detectar famílias em risco de insegurança alimentar (IBGE, 2006). Considera-se que a medida direta de segurança alimentar por meio da EBIA seja um importante indicador para monitoramento da iniquidade, podendo complementar um conjunto de indicadores sociais ou, mesmo de forma isolada, identificar grupos com vulnerabilidade social (PANIGASSI et al., 2008). Esse fato adquire importância na medida em que uma melhor compreensão da natureza dinâmica da insegurança alimentar, além da compreensão dos três componentes básicos (disponibilidade, acesso e utilização biológica dos alimentos), deve incluir análises de subsistência, da vulnerabilidade e das estratégias de gestão de risco (ROSE, 2008).

O nível de segurança alimentar familiar através da EBIA é determinado por meio da quantificação do total de respostas afirmativas na escala: (a) Segurança Alimentar: 0 resposta positiva; (b) Insegurança Alimentar Leve: 1 - 5 respostas positivas; (c) Insegurança Alimentar Moderada: 6 - 10 respostas positivas; (d) Insegurança Alimentar Grave: 11 - 15 respostas positivas (SEGALL-CORRÊA et al., 2004).

Um dos elementos chave do enfoque da EBIA é o conceito de "estratégias ou mecanismos de adaptação" que os lares usam para enfrentar as dificuldades relacionadas com os alimentos. Esses mecanismos podem ser classificados segundo sua frequência (níveis de segurança/insegurança alimentar) e sua severidade (níveis de severidade de insegurança alimentar), que está relacionado ao custo subjetivo, representado em pesos diferentes para as perguntas da escala que a estratégia representa para a família (HOODINOTT, 2003). Baseado nisso, a medida níveis de severidade de insegurança alimentar é proposta para complementar a análise dos resultados da EBIA. A medida almeja reforçar o monitoramento da iniquidade com uso da EBIA, auxiliando, principalmente, na identificação dos grupos para os quais os recursos deverão ser prioritariamente dirigidos. Com o indicador de Severidade da Insegurança Alimentar, os autores pretendem fornecer um instrumento de uso local para identificação de vulnerabilidades associadas à insegurança alimentar.

\section{Metodologia}

Trata-se de um estudo epidemiológico de delineamento transversal, que investigou famílias com crianças menores de cinco anos assistidas em creches cadastradas na Secretaria de Estado do Desenvolvimento Humano do Governo da Paraíba. No contexto familiar, a definição do grupo populacional esteve baseada na vulnerabilidade socioeconômica e na presença de crianças menores de cinco anos. Assim, enfatiza-se a conjuntura da desigualdade social na insegurança alimentar e 
a importância de indicadores do estado nutricional de crianças na medição da segurança alimentar.

Ao todo funcionam 45 creches situadas, geralmente, em áreas carentes dos oito municípios paraibanos beneficiados: João Pessoa (30 creches), Campina Grande (nove creches), além das cidades de Areia, Bayeux, Mamanguape, Itaporanga, Soledade e Umbuzeiro (cada uma delas com uma creche). $\mathrm{O}$ universo de estudo é de 4000 crianças beneficiadas, entre 6-72 meses de idade, estando aproximadamente 2800 no município de João Pessoa, 750 no município de Campina Grande e 450 nos outros municípios.

Foi selecionada uma amostra probabilística em duas etapas. Para garantir a representatividade dos municípios, o sistema de referência para a primeira etapa de amostragem foi ordenado segundo estratos (João Pessoa, Campina Grande, outros municípios), possibilitando a obtenção de um tamanho amostral apropriado para cada estrato. Considerou-se também o porte da creche, isto é, o número de crianças por creche. Na segunda etapa, foram sorteadas, nas 14 creches selecionadas de forma aleatória na primeira etapa, as crianças a serem avaliadas. A opção para determinar o tamanho da amostra do estudo foi a descrição de proporções: $\mathrm{n}=\frac{\mathrm{N} * \mathrm{Z}_{\alpha}^{2} * p^{*} q}{\mathrm{~d}^{2} *(\mathrm{~N}-1)+\mathrm{Z}_{\alpha}^{2} * p^{*} q}$, sendo $\mathrm{N}$ o total da população, $\mathrm{Za}^{2}=1,96^{2}$ (quando a confiança é do $95 \%$ ), p a proporção esperada, $\mathrm{q}=1$ - $\mathrm{p}, \mathrm{d}$ a precisão arbitrária (erro de estimação). Considerou-se $p=10,5 \%$ (crianças com déficit de estatura no Brasil) (SOCIEDADE CIVIL BEM-ESTAR FAMILIAR NO BRASIL, 1999) e $d=3 \%$, totalizando 365 crianças que foram selecionadas por sorteio simples no momento do trabalho de campo.

Os procedimentos para a coleta dos dados foram estabelecidos considerando o seguinte modelo de classificação das variáveis independentes do estudo:

- características biológicas das crianças: sexo, idade, peso/idade, estatura/idade, peso/estatura;

- morbidade e amamentação das crianças: hospitalização nos 12 meses anteriores à entrevista, presença de morbidade nos 15 dias anteriores à entrevista (baseada na referência materna sobre a ocorrência de diarreia, febre, vômitos, tosse e verminose), amamentação ( $\operatorname{sim}$ ou não), tempo de amamentação total, tempo de amamentação exclusivo;

- antecedentes maternos: idade, estatura/idade, Índice de Massa Corporal (IMC), cuidados durante a gravidez baseado em condições associadas à gravidez (orientação sobre a amamentação, suplementação com sulfato ferroso, vacina antitetânica, número de consultas de pré-natal, uso de cigarro, uso de álcool);

- condições socioeconômicas: tipo de casa, número de cômodos do domicílio, número de pessoas no domicílio, renda familiar per capita, benefício do Programa Bolsa Família;

Os dados socioeconômicos, por questão de factibilidade, e considerando sua atualização a cada ato de matrícula da criança, foram obtidos da ficha da criança. Esse documento é obrigatório nas creches e informa sobre o perfil das crianças beneficiárias. As informações sobre morbidade e amamentação da criança, cuidados durante a gravidez e benefício do Programa Bolsa Família, foram obtidas através de questionário pré-testado aplicado às mães das crianças.

Para a avaliação antropométrica, as medidas de comprimento (crianças menores de 24 meses) e a estatura (crianças de $25-72$ meses), assim como o peso e a estatura da mãe, foram obtidas utilizando equipamentos e técnicas padronizadas. Foram obedecidos os procedimentos recomendados pela Organização Mundial da Saúde (OMS) (WORLD HEALTH ORGANIZATION, 1995).

Para a avaliação do estado nutricional das crianças, foram considerados os índices peso/idade, comprimento/idade (estatura/ idade), peso/comprimento (peso/estatura), os quais foram comparados com os padrões de crescimento infantil da OMS (DE ONIS et al., 2004), utilizando o programa WHO Anthro 2005 versão beta (WHO Anthro, Genebra, Suíça). Foram consideradas com déficit nutricional todas as crianças que apresentaram índices com dois escores $\mathrm{z}$ abaixo do valor mediano da população de referência $(<-2$ escores z como ponto de corte para classificar déficit nutricional). Os índices peso/idade e peso/estatura com dois escores $\mathrm{z}$ acima do valor mediano da população de referência foram considerados para indicar peso ele- 
vado para a idade e sobrepeso/obesidade, respectivamente.

Para a avaliação do estado nutricional das mães, foram considerados os índices estatura/idade e IMC. A baixa estatura materna foi definida pelo ponto de corte $155,0 \mathrm{~cm}$ (WORLD HEALTH ORGANIZATION, 2007; NATIONAL CENTER FOR HEALTH STATISTICS, 2000). Para o caso do IMC, foram utilizados os pontos de corte indicados pela OMS (WORLD HEALTH ORGANIZATION, 1995).

A avaliação da segurança alimentar foi realizada utilizando a EBIA. Esse instrumento consta de 15 perguntas centrais fechadas, com opção de respostas sim ou não, sobre a experiência nos últimos três meses de insuficiência alimentar em diferentes níveis de intensidade. Inclui desde a preocupação de que a comida possa acabar até a vivência de passar um dia todo sem comer (SALLES-COSTA et al., 2008).

A medida níveis de severidade de insegurança alimentar foi desenhada para os fins propostos. É uma medida que considera somente a população com algum grau de insegurança alimentar por meio da quantificação do total de respostas afirmativas na EBIA. As famílias foram classificadas em três categorias de insegurança alimentar considerando a medida níveis de severidade de insegurança alimentar: (a) Severidade de Insegurança Alimentar Grau I: 1 - 10 pontos; (b) Severidade de Insegurança Alimentar Grau II: 11 - 20 pontos; (c) Severidade de Insegurança Alimentar Grau III: 21 - 30 pontos. Para isso: i) cada resposta positiva equivaleu a um ponto; ii) as perguntas da EBIA foram classificadas por categorias e atribuídos pesos diferentes correspondentes à severidade, denotando a reação das famílias em consequência da insuficiência alimentar que é expressa na pergunta (perguntas 2-4 que apontam a deficiência de acesso à alimentação: peso 1, perguntas 5-10 que apontam sobre o comprometimento da qualidade da dieta: peso 2, perguntas 11 - 15 que apontam a redução quantitativa da alimentação: peso 3). Nenhum peso foi atribuído à primeira pergunta da EBIA, pois ela manifesta somente preocupação ante a escassez de alimentos, e não propriamente um mecanismo de adaptação.

Os dados foram codificados e digitados em planilhas do programa Excel (Microsoft
Inc., Estados Unidos), por dois digitadores independentes para verificação e correção de erros de digitação. $\mathrm{O}$ aplicativo Validate do programa Epi Info v. 6.04b (WHO/CDC, Atlanta, Estados Unidos) foi usado para analisar a consistência dos dados.

Para verificar a existência de associação entre os níveis de severidade de insegurança alimentar com as variáveis independentes do modelo de estudo, foi utilizado o teste quiquadrado de Pearson. O nível de significância estatística considerado foi de $5 \%(p<0,05, a=$ $0,05)$. Em seguida, realizou-se análise multivariada em níveis segundo as categorias de classificação das variáveis, ajustando os modelos pelas variáveis associadas significativamente ao nível de $5 \%$. As variáveis que permaneceram associadas em cada nível constituíram o modelo hierárquico final. As análises não foram ajustadas por sexo e idade da criança por não apresentarem diferença significativa no modelo univariado ( $p>0,05)$.

A confiabilidade ou consistência interna da EBIA foi analisada por meio do coeficiente a-Cronbach, utilizando como valores aceitáveis resultados acima de 0,85 . As análises estatísticas foram realizadas utilizando-se o programa SPSS for Windows versão 17.0 (SPSS Inc., Chicago, Estados Unidos).

Os resultados do presente estudo fazem parte do projeto aprovado pelo Comitê de Ética em Pesquisa da Universidade Estadual da Paraíba, protocolado sob n. 4233000013307, intitulado "Segurança Alimentar e Nutricional de famílias socioeconomicamente vulneráveis do estado da Paraíba, Brasil: explorando associações entre medidas essenciais de segurança alimentar". No momento da entrevista, foi apresentado o termo de consentimento, no qual o entrevistado afirmava a concordância em participar da pesquisa, após esclarecimentos quanto aos procedimentos a serem empregados, a garantia de sigilo das informações prestadas e a possibilidade de se recusar a participar da pesquisa.

\section{Resultados}

Da amostra de 365 crianças, registrou-se um total de 33 perdas $(9,04 \%)$, ocasionadas pelo não comparecimento das mães ou pessoas responsáveis pelas crianças no momento da coleta de dados ou por questionários da EBIA 
incompletos. A fim de analisar a possibilidade de viés da amostra, foi comparada a média de idade e a proporção por sexo da amostra final com a amostra das crianças que foram perdidas, não diferindo entre os dois grupos. Isso permite extrapolar os resultados obtidos para o total da população. Nas 332 famílias avaliadas, 133 delas foram classificadas com segurança alimentar, restando 199 para a avaliação da severidade de insegurança alimentar. O teste estatístico $\alpha$-Cronbach indicou a consistência e confiabilidade dos resultados, com validade psicométrica alta $(0,9206)$.

Nos domićlios com insegurança alimentar, $53,8 \%$ das crianças eram do sexo masculino, 63,8\% tinham entre 37-60 meses, 7,5\% apresentavam desnutrição crônica e 7,5\% sobrepeso/obesidade. Em relação aos dados maternos, 8,5\% tinham menos de 20 anos, $39,7 \%$ baixa estatura, $35,7 \%$ sobrepeso/obesidade, e $31,7 \%$ das entrevistadas referiram cuidados inadequados durante a gravidez. Foi verificado que $19,1 \%$ dos domicílios tinham menos de quatro cômodos e $29,1 \%$, seis pessoas ou mais. Além disso, a maioria das famílias $(94,5 \%)$ tinha renda per capita inferior a $1 \frac{1}{2}$ salário mínimo e $45,7 \%$ eram beneficiários do Programa Bolsa Família.

A severidade de insegurança alimentar grau I foi registrada em $62,8 \%$ das famílias, enquanto $19,6 \%$ e $17,6 \%$ das famílias foram classificadas nas categorias severidade de insegurança alimentar grau II e severidade de insegurança alimentar grau III, respectivamente.

A distribuição dos níveis de severidade de insegurança alimentar segundo variáveis biológicas das crianças, morbidade e amamentação das crianças, antecedentes maternos e condições socioeconômicas, é apresentada na tabela 1. Ser beneficiário do Programa Bolsa Família, baixa estatura materna e idade da mãe $<20$ anos foram fatores associados à severidade de insegurança alimentar. Mães com inadequados cuidados durante a gravidez também contribuiu negativamente $(\mathrm{p}=0,013)$ com a severidade de insegurança alimentar.

Os resultados da tabela 2 apresentam os valores da Razão de Chances e Intervalo de Confiança de 95\% dos determinantes da severidade de insegurança alimentar em modelo ajustado. A tabela mostra como os graus mais severos de insegurança alimentar tiveram maior probabilidade de ocorrência nas famílias beneficiárias do Programa Bolsa Família, de mães com baixa estatura, e de mães com cuidados inadequados durante a gravidez. As famílias de mães com baixa estatura apresentaram três vezes mais chance de ter severidade de insegurança alimentar grau II e III quando comparadas com as famílias de mães com estatura normal. Considerando a participação das famílias no Programa Bolsa Família, aquelas beneficiadas pelo programa tiveram 2,04 mais chances de ter maior severidade de insegurança alimentar. Cuidados inadequados durante a gravidez também foi um fator associado a graus de severidade de insegurança alimentar grau II e III, aumentando a chance em 1,98.

\section{Discussão}

O instrumento utilizado nesta pesquisa para avaliar a segurança alimentar tem ganhado reconhecimento internacional como ferramenta importante para a medida da dimensão de acesso aos alimentos. A escala reflete três avanços conceituais importantes para a medida de segurança alimentar e nutricional: i) menos ênfase no uso de indicadores de disponibilidade e utilização de alimentos e mais no uso de medidas de acesso aos alimentos; (2) mudança de enfoque nas medidas objetivas para as subjetivas; e (3) ênfase crescente na mensuração fundamental (direta) em vez de medidas indiretas. Além disso, argumenta-se sua capacidade de contemplar não apenas a dificuldade de acesso familiar aos alimentos, mas também as dimensões psicológicas e sociais da insegurança alimentar (KEPPLE; SEGALL-CORRÊA, 2011).

A adoção de um instrumento único para avaliar a insegurança alimentar, nacionalmente aceito, como é a EBIA no Brasil, possibilita a obtenção de resultados padronizados que permitem um melhor mapeamento, identificação de grupos vulneráveis e compreensão dos fatores determinantes. As questões da EBIA incluem a preocupação e a ansiedade da família em relação à obtenção dos alimentos, perpassando pelo comprometimento da qualidade e quantidade da dieta de adultos e crianças, e alcançando situações mais graves, com restrições quantitativas de alimentos para todos os membros da família 
(KEPPLE; SEGALL-CORRÊA, 2011; SEGALLCORRÊA et al., 2004). Sendo assim, um dos elementos chave deste enfoque está nos mecanismos de adaptação ou estratégias usadas para enfrentar as dificuldades relacionadas com a alimentação. Esses mecanismos podem ser classificados considerando sua frequência de uso e sua severidade. A severidade representa o rigor do mecanismo de adaptação adotado (SEGALL-CORRÊA et al., 2004; HOODINOTT, 2003).

A EBIA tem sido utilizada no Brasil em diferentes pesquisas locais e nacionais de forma satisfatória. Porém as medições realizadas têm considerado somente a frequência das respostas afirmativas (MELGASQUINONEZ; HACKETT, 2008). Pioneira é a experiência deste estudo para medir a severidade de insegurança alimentar. Tanto em termos de tendência esperada das categorias das variáveis como na significância estatística das razões de chance, os resultados obtidos não diferem muito dos resultados da medida dos níveis de segurança/insegurança alimentar verificada com a quantificação do total de respostas positivas (FIGUEROA PEDRAZA et al., no prelo). Esse fato pode ser utilizado como parâmetro de validade da medida de severidade de insegurança alimentar.

A plausibilidade das significâncias estatísticas encontradas também pode ser usada para reforçar a validez dos resultados. Neste estudo, as mães de baixa estatura, as mães com cuidados inadequados durante a gravidez e o benefício do Programa Bolsa Família são as que melhor caracterizam o maior risco associado às consequências negativas de problemas no acesso aos alimentos. A baixa estatura materna constitui um fator de contexto social relacionado com o rendimento produtivo e físico e com o estado de saúde, podendo, então, afetar a capacidade econômica e de prestar cuidados. Por sua vez, os cuidados durante a gravidez podem expressar a preocupação com o bem-estar da criança (UNITED NATIONS, 2000). A importância da condição socioeconômica como determinante da insegurança alimentar, apontada pelo benefício do Programa Bolsa Família nos resultados do presente estudo, também tem sido observada em outros estudos (PANIGASSI, 2005; SALLES-COSTA et al., 2008). Possibilita-se, assim, explicar as maiores chances de níveis de severidade de insegurança alimentar grau II e III nas famílias de mães com baixa estatura, nas famílias de mães com cuidados inadequados durante a gravidez e nas famílias beneficiarias do Programa Bolsa Família.

A importância de avaliar a severidade de insegurança alimentar fundamenta-se na sua possibilidade de retratar de forma mais completa o risco ou temor sobre a continuidade do acesso aos alimentos, emitindo critério de desigualdade. Isto constitui um elemento de grande utilidade na definição de intervenções efetivas. Se o objetivo de uma determinada intervenção é diminuir a porcentagem de pessoas com insegurança alimentar, a prevalência de insegurança alimentar deve ser a medida usada como indicador. Porém, se o objetivo é atingir as pessoas com maior insegurança alimentar, a severidade de insegurança alimentar deve ser a medida considerada. O impacto de uma estratégia sempre será maior sobre a medida de severidade, pois as famílias que utilizam mecanismos de adaptação mais severos devem ser mais vulneráveis. A severidade pode favorecer o estabelecimento de metas de cobertura e a identificação dos grupos para os quais os recursos das políticas públicas devem ser prioritariamente dirigidos. Além disso, ao ponderar pesos diferentes para os diversos mecanismos de adaptação, a severidade possibilita focalizar os indivíduos com maior risco das consequências negativas associadas a problemas de acesso aos alimentos como a morbimortalidade e o déficit de crescimento (HOODINOTT, 2003; MALETTA; GÓMEZ, 2004).

Do ponto de vista de focalização das políticas sociais, a severidade de insegurança alimentar pode contribuir nos seguintes aspectos argumentados na literatura:

- Um programa integrado de segurança alimentar deve atentar para os três elementos mencionados que dizem respeito ao acesso aos alimentos: quantidade suficiente, regularidade e qualidade. Dessa maneira, a questão não é apenas elevar a renda das pessoas pobres, mas, também, garantir que essa renda seja utilizada para o consumo de alimentos (BELIK, 2003).

Uma vez que: i) a segurança alimentar e nutricional é determinada por múltiplos 
fatores; ii) as famílias possuem diferentes gradientes de vulnerabilidade com características e contextos de vida diferentes; iii) existe uma lógica inserida na escolha e o perfil de gastos familiares; a construção de políticas públicas integradas requer a combinação das informações de renda com outros indicadores sociais, de saúde e nutrição, isso torna possível a adequação das intervenções às características familiares, o que é, em grande medida, decisivo no seu potencial (BURLANDY, 2007).

O estabelecimento de mecanismos de focalização eficientes, também lido como equidade, apresenta importantes desafios. O déficit de cobertura de muitos programas costuma afetar mais gravemente aqueles grupos mais vulneráveis aos quais os programas são dirigidos, comprometendo o combate à iniquidade (BURLANDY, 2007; FONSECA; VIANA, 2007; LAVINAS, 2007).

\section{Considerações finais}

O nível de severidade de insegurança alimentar representa um indicador direto das desigualdades sociais. Esse indicador possibilita identificar, entre os indivíduos com insegurança alimentar, aqueles com maior vulnerabilidade. O conhecimento dos níveis de severidade de insegurança alimentar pode contribuir para o êxito de intervenções na área de segurança alimentar e de combate à desnutrição infantil na medida em que possibilita o direcionamento de recursos para aqueles grupos populacionais nos quais as estratégias de intervenção devem ser prioritárias. As iniquidades sociais em saúde devem, assim, ser impactadas positivamente, uma vez que a segurança alimentar está relacionada a diferentes problemáticas de saúde, ao crescimento e desenvolvimento cognitivo de crianças, assim como com a capacidade de socialização.

Tabela 1 - Prevalência de severidade de insegurança alimentar familiar segundo características biológicas das crianças, morbidade e amamentação das crianças, antecedentes maternos e condições socioeconômicas. Paraíba, 2008.

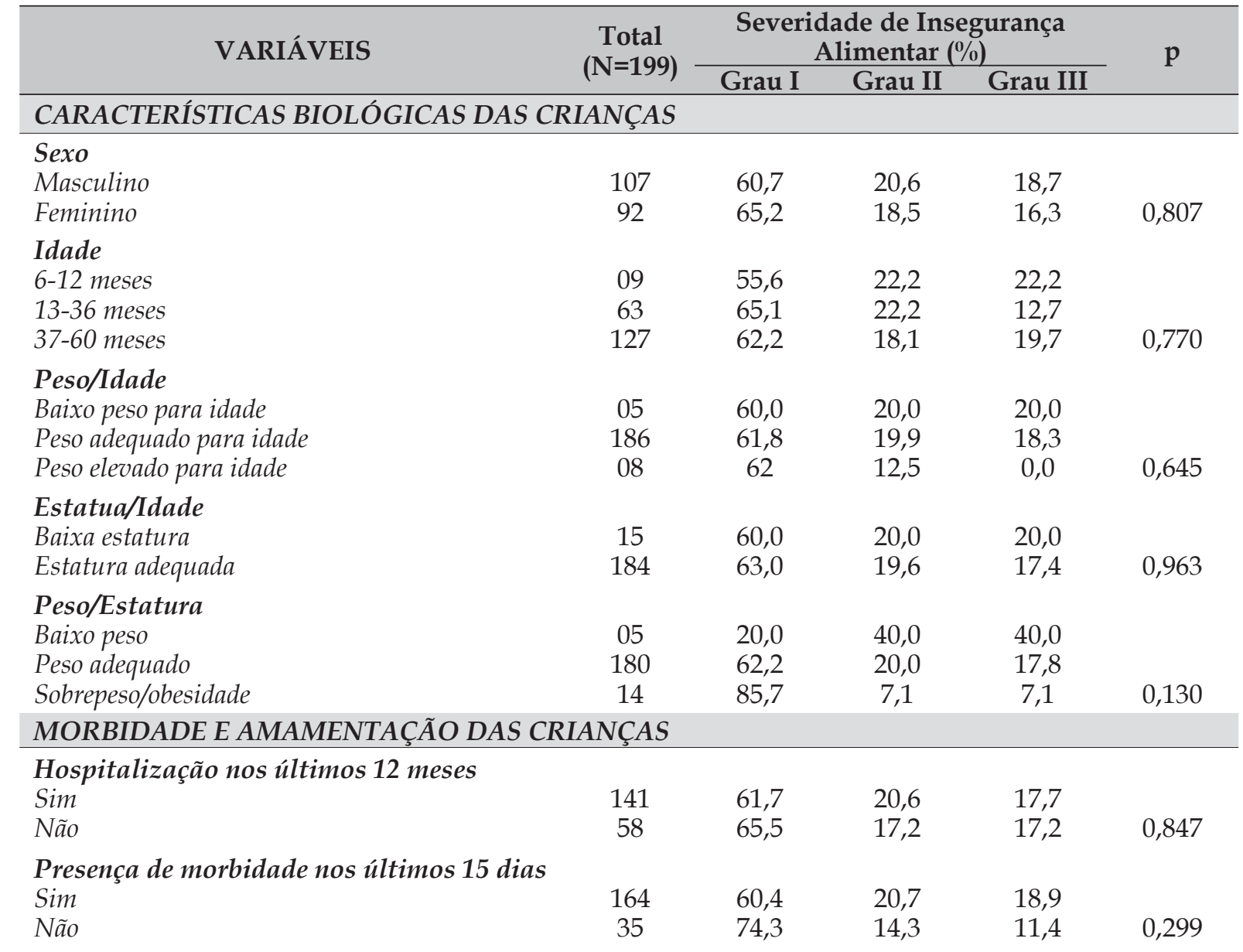




\begin{tabular}{|c|c|c|c|c|c|}
\hline \multirow[t]{2}{*}{ VARIÁVEIS } & \multirow{2}{*}{$\begin{array}{c}\text { Total } \\
(\mathrm{N}=199)\end{array}$} & \multicolumn{3}{|c|}{$\begin{array}{l}\text { Severidade de Insegurança } \\
\text { Alimentar (\%) }\end{array}$} & \multirow[t]{2}{*}{ p } \\
\hline & & Grau I & Grau II & Grau III & \\
\hline $\begin{array}{l}\text { Amamentação } \\
\text { Sim } \\
\text { Não } \\
\text { Sem informação }\end{array}$ & $\begin{array}{l}188 \\
10 \\
01\end{array}$ & $\begin{array}{c}62,8 \\
60,0 \\
100,0\end{array}$ & $\begin{array}{l}19,7 \\
20,0 \\
0,0\end{array}$ & $\begin{array}{c}17,6 \\
20,0 \\
0,0\end{array}$ & 0,958 \\
\hline $\begin{array}{l}\text { Tempo de amamentação total } \\
<24 \text { meses } \\
\geq 24 \text { meses } \\
\text { Sem informação }\end{array}$ & $\begin{array}{l}151 \\
46 \\
02\end{array}$ & $\begin{array}{l}67,5 \\
47,8 \\
50,0\end{array}$ & $\begin{array}{l}15,2 \\
32,6 \\
50,0\end{array}$ & $\begin{array}{c}17,2 \\
19,6 \\
0,0\end{array}$ & 0,060 \\
\hline $\begin{array}{l}\text { Tempo de amamentação exclusivo } \\
<6 \text { meses } \\
\geq 6 \text { meses } \\
\text { Sem informação }\end{array}$ & $\begin{array}{l}125 \\
70 \\
04\end{array}$ & $\begin{array}{l}68,0 \\
55,7 \\
25,0\end{array}$ & $\begin{array}{l}16,0 \\
25,7 \\
25,0\end{array}$ & $\begin{array}{l}16,0 \\
18,6 \\
50,0\end{array}$ & 0,149 \\
\hline ANTECEDENTES MATERNOS & & & & & \\
\hline $\begin{array}{l}\text { Idade da mãe (anos) } \\
\geq 30 \\
<30-\geq 25 \\
>25-\geq 20 \\
<20\end{array}$ & $\begin{array}{l}64 \\
57 \\
58 \\
17\end{array}$ & $\begin{array}{l}64,0 \\
59,6 \\
67,2 \\
47,0\end{array}$ & $\begin{array}{c}26,6 \\
24,6 \\
12,1 \\
5,9\end{array}$ & $\begin{array}{l}9,4 \\
15,8 \\
20,7 \\
47,1\end{array}$ & 0,012 \\
\hline $\begin{array}{l}\text { Estatura/Idade } \\
\text { Baixa estatura } \\
\text { Estatura normal } \\
\text { Sem informação }\end{array}$ & $\begin{array}{c}79 \\
112 \\
08\end{array}$ & $\begin{array}{l}48,1 \\
74,1 \\
50,0\end{array}$ & $\begin{array}{c}35,4 \\
8,9 \\
12,5\end{array}$ & $\begin{array}{l}16,5 \\
17,0 \\
37,5\end{array}$ & $<0,001$ \\
\hline $\begin{array}{l}\text { IMC } \\
\text { Obesidade } \\
\text { Sobrepeso } \\
\text { Normalidade } \\
\text { Baixo peso } \\
\text { Sem informação }\end{array}$ & $\begin{array}{l}17 \\
54 \\
105 \\
15 \\
08\end{array}$ & $\begin{array}{l}64,7 \\
59,3 \\
63,8 \\
73,4 \\
50,0\end{array}$ & $\begin{array}{l}29,4 \\
24,1 \\
17,1 \\
13,3 \\
12,5\end{array}$ & $\begin{array}{l}5,9 \\
16,7 \\
19,0 \\
13,3 \\
37,5\end{array}$ & 0,618 \\
\hline $\begin{array}{l}\text { Cuidados durante a gravidez } \\
\text { Adequados } \\
\text { Inadequados } \\
\text { Sem informação }\end{array}$ & $\begin{array}{l}131 \\
63 \\
05\end{array}$ & $\begin{array}{c}67,2 \\
50,8 \\
100,0\end{array}$ & $\begin{array}{c}20,6 \\
19,0 \\
0,0\end{array}$ & $\begin{array}{c}12,2 \\
30,2 \\
0,0\end{array}$ & 0,013 \\
\hline CONDIÇÕES SOCIOECONÔMICAS & & & & & \\
\hline $\begin{array}{l}\text { Tipo de casa } \\
\text { Própria } \\
\text { Alugada } \\
\text { Cedida } \\
\text { Invadida }\end{array}$ & $\begin{array}{l}104 \\
70 \\
19 \\
06\end{array}$ & $\begin{array}{l}62,5 \\
68,6 \\
52,6 \\
33,3\end{array}$ & $\begin{array}{l}24,0 \\
11,4 \\
26,3 \\
16,7\end{array}$ & $\begin{array}{l}13,5 \\
20,0 \\
21,1 \\
50,0\end{array}$ & 0,110 \\
\hline $\begin{array}{l}\text { Número de cômodos do domicílio } \\
<4 \\
\geq 4\end{array}$ & $\begin{array}{c}38 \\
161\end{array}$ & $\begin{array}{l}47,4 \\
66,5\end{array}$ & $\begin{array}{l}28,9 \\
17,4\end{array}$ & $\begin{array}{l}23,7 \\
16,1\end{array}$ & 0,087 \\
\hline $\begin{array}{l}\text { Número de pessoas no domicílio } \\
\geq 6 \\
<6\end{array}$ & $\begin{array}{c}58 \\
141\end{array}$ & $\begin{array}{l}67,2 \\
61,0\end{array}$ & $\begin{array}{l}15,5 \\
21,3\end{array}$ & $\begin{array}{l}17,2 \\
17,7\end{array}$ & 0,620 \\
\hline $\begin{array}{l}\text { Renda familiar per capita }{ }^{2} \\
\geq 2 S M \\
<2 S M-\geq 1 S M \\
<1 S M-\geq 1 / 2 S M \\
<1 / 2 S M \\
\text { Sem informação }\end{array}$ & $\begin{array}{c}00 \\
01 \\
12 \\
184 \\
02\end{array}$ & $\begin{array}{c}0,0 \\
100,0 \\
91,7 \\
60,3 \\
100,0\end{array}$ & $\begin{array}{c}0,0 \\
0,0 \\
8,3 \\
20,7 \\
0,0\end{array}$ & $\begin{array}{c}0,0 \\
0,0 \\
0,0 \\
19,0 \\
0,0\end{array}$ & 0,344 \\
\hline $\begin{array}{l}\text { Benefício do Programa Bolsa Família } \\
\text { Não recebe } \\
\text { Beneficiário }\end{array}$ & $\begin{array}{c}108 \\
91\end{array}$ & $\begin{array}{l}70,4 \\
53,8\end{array}$ & $\begin{array}{l}13,0 \\
27,5\end{array}$ & $\begin{array}{l}16,7 \\
18,7\end{array}$ & 0,028 \\
\hline
\end{tabular}

${ }^{1}$ Foram consideradas mães com cuidados adequados durante a gravidez aquelas que receberam orientação sobre a amamentação, suplementação com sulfato ferroso, vacina antitetânica, três ou mais consultas pré-natais e que não fizeram uso de cigarro nem de álcool durante o período gestacional.

${ }^{2}$ Considerando o valor do salário mínimo da época $(\mathrm{R} \$ 416,00)$.

IMC: Índice de Massa Corporal. SM: Salário Mínimo.

p: valores de p para o teste estatístico qui-quadrado; $\mathrm{p}$ em negrito denota significância estatística. 
Tabela 2 - Medidas de Risco (Razão de Chances - RC) e Intervalo de Confiança (IC95\%) para os níveis de severidade de insegurança alimentar segundo as variáveis com significância estatística. Paraíba, 2008.

\begin{tabular}{|c|c|c|c|c|}
\hline Variáveis & $\begin{array}{c}\text { Insegurança } \\
\text { Alimentar Grau I }\end{array}$ & $\begin{array}{c}\text { Insegurança Alimentar } \\
\text { Grau II e III }\end{array}$ & $\mathrm{RC}$ & IC95\% \\
\hline \multicolumn{5}{|l|}{ Idade da mãe (anos) } \\
\hline$>=20$ & 114 & 65 & 1 & \\
\hline$<20$ & 08 & 9 & 1,97 & $0,73-5,36$ \\
\hline \multicolumn{5}{|c|}{ Estatura/Idade da mãe } \\
\hline Estatura normal & 83 & 29 & 1 & \\
\hline Baixa estatura & 38 & 41 & 3,09 & $1,68-5,69$ \\
\hline \multicolumn{5}{|c|}{ Cuidados durante a gravidez ${ }^{1}$} \\
\hline Adequados & 88 & 43 & 1 & \\
\hline Inadequados & 32 & 31 & 1,98 & $1,07-3,66$ \\
\hline \multicolumn{5}{|c|}{$\begin{array}{l}\text { Benefício do Programa Bolsa } \\
\text { Família }\end{array}$} \\
\hline Não recebe & 76 & 49 & 1 & \\
\hline Beneficiário & 32 & 42 & 2,04 & $1,14-3,65$ \\
\hline
\end{tabular}

${ }^{1}$ Foram consideradas mães com cuidados adequados durante a gravidez aquelas que receberam orientação sobre a amamentação, suplementação com sulfato ferroso, vacina antitetânica, três ou mais consultas pré-natais e que não fizeram uso de cigarro nem de álcool durante o período gestacional.

IC95\% em negrito denota associação estatística.

\section{Referências}

BELIK, W. Perspectivas para segurança alimentar e nutricional no Brasil. Saúde e Sociedade, São Paulo, v. 12, n. 1, p. 12-20, 2003.

BURLANDY, L. Transferência condicionada de renda e segurança alimentar e nutricional. Ciência E Saúde Coletiva, Rio de Janeiro, v. 12, n. 6, p. 1441-51, 2007.

DE ONIS, M. et al. Measurement and standardization protocols for anthropometry used in the construction of a new international growth reference. Food and Nutrition Bulletin, Boston, USA, v. 25, p. 15-27, 2004. Suplemento.

FAVARO, T. et al. Segurança alimentar em famílias indígenas Terena, Mato Grosso do Sul, Brasil. Cadernos de Saúde Pública, Rio de Janeiro, v. 23, n. 4, p. 785-93, 2007.

FIGUEROA PEDRAZA, D. et al. Segurança alimentar em famílias com crianças matriculadas em creches públicas do estado da Paraíba, Brasil. Revista de Nutrição, no prelo 2013.

FONSECA, A. M. M.; VIANA, A. L. A. Direito à saúde, atenção básica e transferências condicionadas de renda na América Latina. Ciência E Saúde Coletiva, Rio de Janeiro, v. 12, n. 6, p. 1505-12, 2007.

HOODINOTT, J. Escogiendo indicadores de resultado de la seguridad alimentaria del hogar. Instituto Internacional de Investigación sobre Políticas Alimentarias. In: HOODINOTT, J. (Ed.). Seguridad Alimentaria en la Práctica: Métodos para Proyectos de Desarrollo Rural. Washington, DC: IFPRI, 2003.

INSTITUTO BRASILEIRO DE GEOGRAFIA E ESTATÍSTICA - IBGE. Pesquisa Nacional por Amostra de Domicílios (PNAD) 2004: segurança alimentar. Rio de Janeiro: IBGE, 2006.
KEPPLE, A. W.; SEGALL-CORRÊA, A. M. Conceituando e medindo segurança alimentar e nutricional. Ciência E Saúde Coletiva, Rio de Janeiro, v. 16, n. 1 , p. 187-99, 2011.

LAVINAS, L. Gasto social no Brasil: programas de transferência de renda versus investimento social. Ciência $\mathcal{E}$ Saúde Coletiva, Rio de Janeiro, v. 12, n. 6, p. 1463-76, 2007.

MALETTA, H.; GÓMEZ, R. Seguridad Alimentaria: medición y métodos. Roma: FAO, 2004.

MELGAS-QUINONEZ, H.; HACKETT, M. Measuring household food security: the global experience. Revista de Nutrição, Campinas, v. 21, p. 27-37, 2008. Suplemento.

NATIONAL CENTER FOR HEALTH STATISTICS. Centers for Disease Control and Prevention. National Health and Nutrition Examination Survey. USA: CDC, 2000. Disponível em: <http://www.cdc.gov/growtharts>. Acesso em: 13 mar. 2010.

PANELLI-MARTINS, B. E.; SANTOS, S. M. C.; ASSIS, A. M. O. Segurança alimentar e nutricional: desenvolvimento de indicadores e experimentação em um município da Bahia, Brasil. Revista de Nutrição, Campinas, v. 21, p. 65-81, 2008. Suplemento.

PANIGASSI, G. et al. Insegurança alimentar como indicador de iniqüidade: análise de inquérito populacional. Cadernos de Saúde Pública, Rio de Janeiro, v. 24, n. 10, p. 2376-84, 2008.

PANIGASSI, G. Inquérito populacional sobre a percepção da segurança alimentar intrafamiliar no município de Campinas, SP. Campinas, 2005. Tese (Doutorado em Saúde Coletiva) - Universidade Estadual de Campinas, Campinas, 2005.

PEREIRA, R. A.; SANTOS, L. M. P. A dimensão da insegurança alimentar. Revista de Nutrição, Campinas, v. 21, p. 7-13, 2008. Suplemento. 
PÉREZ-ESCAMILLA, R.; SEGALL-CORRÊA, A. M. Food insecurity measurement and indicators. Revista de Nutrição, Campinas, v. 21, p. 15-26, 2008. Suplemento.

PRADO, S. D. et al. A pesquisa sobre segurança alimentar e nutricional no Brasil de 2000 a 2005: tendências e desafios. Ciência \& Saúde Coletiva, Rio de Janeiro, v. 15, n. 1, p. 7-18, 2010.

ROSE, D. D. Interventions to reduce household food insecurity: a synthesis of current concepts and approaches for Latin America. Revista de Nutrição, Campinas, v. 21, p. 159-73, 2008. Suplemento.

SALLES-COSTA, R. et al. Associação entre fatores socioeconômicos e insegurança alimentar: estudo de base populacional na região metropolitana do Rio de Janeiro, Brasil. Revista de Nutrição, Campinas, v. 21, p. 99-109, 2008. Suplemento.

SEGALL-CORREAA, A. M. et al. Acompanhamento e avaliação da Segurança Alimentar de famílias brasileiras: validação de metodologia e de instrumento de coleta de informação: Urbano/Rural, 2004. Campinas: Universidade Estadual de Campinas, 2004. Disponível em: <http:// www.opas.org.br/sistema/arquivos/vru_unic.pdf>. Acesso em: 7 abr. 2011.

SOCIEDADE CIVIL BEM-ESTAR FAMILIAR NO BRASIL. Programa de Demografia e Saúde. Pesquisa Nacional sobre Demografia e Saúde 1996. 2. ed. Rio de Janeiro, 1999.

UNITED NATIONS. Adninistrative Committee on Coordination Sub-Committee on Nutrition. 4 Report on the World Nutrition Situation: Nutrition throughout the life cycle. Geneva: ACC/SCN, 2000.

URIBE, M. C. A.; RESTREPO, A. E. Inseguridad alimentaria de los hogares colombianos según localización geográfica y algunas condiciones sociodemográficas. Perspectivas en Nutrición Humana, Medellín, Colômbia v. 10, n. 1, p. 23-36, 2008.

WORLD HEALTH ORGANIZATION. Global database on child growth and malnutrition. Geneva: WHO, 2007. Disponível em: <http://www.who.int/nutgrowthdb/ index.html>. Acesso em: 5 mar. 2010.

Physical status: the use and interpretation of anthropometry: report of an expert committee. Geneva: WHO, 1995. Technical Report Series, 854WHO. 